\title{
MURMUR: a new low-noise experiment for the search of neutron-hidden neutron transitions in the context of braneworld scenarios
}

\author{
Coraline Stasser, ${ }^{a, *}$ Guy Terwagne, ${ }^{a}$ Jacob Lamblin, ${ }^{b}$ Olivier Méplan, ${ }^{b}$ Guillaume \\ Pignol, ${ }^{b}$ Bernard Coupé, ${ }^{c}$ Silva Kalcheva, ${ }^{c}$ Steven Van Dyck ${ }^{c}$ and Michaël Sarrazin ${ }^{d}$ \\ ${ }^{a}$ Laboratory for Analysis by Nuclear Reactions, Department of Physics, University of Namur, 61 rue de \\ Bruxelles, B-5000 Namur, Belgium \\ ${ }^{b}$ LPSC, Université Grenoble-Alpes, CNRS/IN2P3, 53 Avenue des Martyrs, F-38026 Grenoble, France \\ ${ }^{c}$ Belgian Nuclear Research Centre (SCK·CEN), Boeretang 200, B-2400 Mol, Belgium \\ 'Institut UTINAM, CNRS/INSU, UMR 6213, Université de Bourgogne-Franche-Comté, 16 route de Gray, \\ F-25030 Besançon Cedex, France \\ E-mail: coraline.stasser@unamur.be, michael.sarrazin@ac-besancon.fr
}

MURMUR is a new passing-through-walls neutron experiment installed near the BR2 nuclear reactor at the Belgian Nuclear Research Center (SCK·CEN, Mol, Belgium) and designed to search neutron interbrane transitions in the context of braneworld scenarios. In such scenarios, our visible universe could be a 3-brane embedded in a multidimensional space-time, called the bulk, which could contain some other invisible adjacent 3-branes. Theoretical works have shown that the existence of hidden braneworlds can be experimentally tested thanks to neutron exchanges between braneworlds. A neutron $n$ in our visible brane can be converted into a hidden neutron $n^{\prime}$ - propagating in a hidden braneworld - when scattered by a nucleus with a cross section $\sigma\left(n \rightarrow n^{\prime}\right) \sim \sigma_{E}(n \rightarrow n) \times p$, where $\sigma_{E}$ is the usual elastic cross-section and $p$ the neutron swapping probability. Hidden neutrons could thus be generated in the moderator of the BR2 nuclear reactor where a high neutron flux undergoes many elastic scatterings. This hidden neutron flux could be free to travel out of the biological shielding of the reactor up to a matrix made of lead, acting as an antenna, which makes possible to regenerate hidden neutrons into visible ones thanks to reverse swapping. These regenerated neutrons can be detected thanks to a neutron detector placed inside the lead block. The MURMUR experimental setup is described, as well as the included upgrades compared to the first experiment of this kind carried out at the ILL (Grenoble, France) in 2015. The first results of MURMUR are introduced and discussed, and some upcoming improvements of the experimental setup are introduced.

40th International Conference on High Energy physics - ICHEP2020

July 28 - August 6, 2020

Prague, Czech Republic (virtual meeting)

\footnotetext{
${ }^{*}$ Speaker
} 


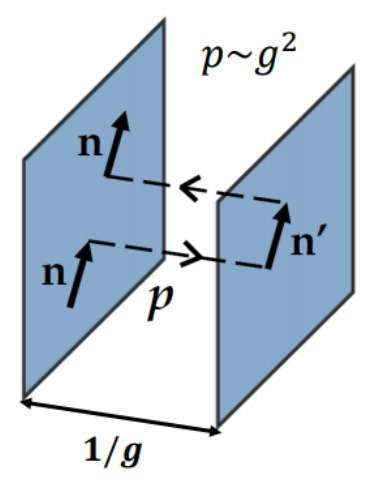

Figure 1: Two adjacent braneworlds modeled by a $M_{4} \times Z_{2}$ non-commutative two-sheeted spacetime. Neutron could leap from our visible state to reach another one in the bulk with a probability $p$ and then come back in our visible state with the same probability $p$. The phenomenology related to $n \rightarrow n^{\prime} \rightarrow n$ transitions is then proportional to $p^{2}$. The studied parameter $g \sim \sqrt{p}$, i.e. the coupling constant between the neutrons in each braneworld, characterizes the geometrical mixing between the visible and the hidden states of the neutron.

\section{Context of the research}

This research is conducted in the context of the hidden sector hypothesis, which is an extension of both the Standard Model of particles (SM) and the $\Lambda \mathrm{CDM}$ cosmological model. The purpose of such hypothesis is to address some questions about dark matter, dark energy or hierarchy and unification problems. The hidden sector can take two different forms. It could be a duplication of the SM content in which each particle of the SM owns a mirror partner. The hidden sector could also have a geometrical origin in the context of braneworld scenarios. In such scenarios, our visible universe is (3+1)-hypersurface (a 3-brane) embedded in a hyperspace (the bulk) with more than $(3+1)$ dimensions. By extension of the Copernican principle, other braneworlds could coexist in the bulk but hidden to us. The research discussed in this paper is a subclass of hidden sector models in which fermions could exist in both the visible and the hidden states. In particular, a neutron $n$ would have a sterile hidden state $n^{\prime}$. Mixings between the two states could occur and lead to $n \leftrightarrow n^{\prime}$ transitions. While braneworlds are often considered in the literature, neutrons could undergo fast oscillations between two adjacent braneworlds with a probability $p$ [1]. Such a phenomenology leads to a new way to probe braneworld scenarios with $n \rightarrow n^{\prime} \rightarrow n$ transitions [2-4]. Fig. 1 summarizes the phenomenology into consideration in the context of braneworld scenarios. The parameter of interest here is $g$, the coupling constant between the neutrons in each braneworld, given by the expression below [1,5]:

$$
g \sim \frac{m^{2}}{M_{B}}
$$

where $M_{B}$ is the energy scale of the branes (TeV, GUT or Planck scale) and $m$ the mass of a constituent quark (340 MeV [5]). The coupling constant $g$, as a phenomenological parameter to constrain the braneworld hypothesis, leads to new physics reachable even at the Planck scale [5].

Neutron disappearance/reappearance toward/from a hidden brane can be induced thanks to nuclei with a high elastic cross section, as summarized in Fig. 2 [2]. When a neutron collides with 


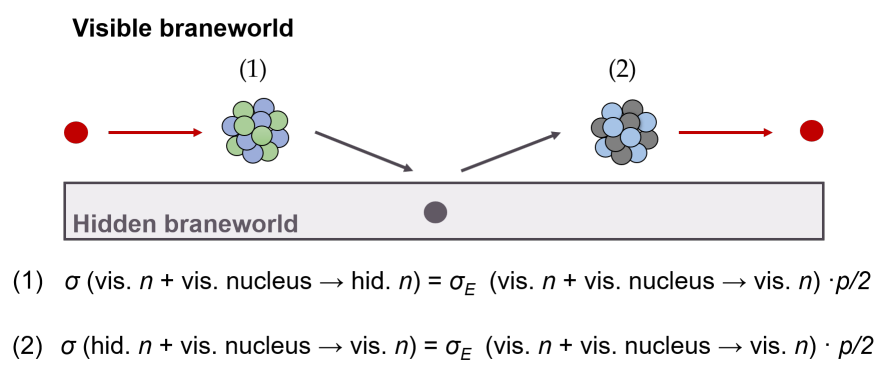

Figure 2: Sketch of the visible neutron - hidden neutron -visible neutron transformation $\left(n \rightarrow n^{\prime} \rightarrow n\right)$ induced by a nucleus with a high elastic cross section.

a nucleus, this one acts as a detector and one has a probability $p$ to measure the neutron in the hidden state and a probability $1-p$ to measure it in our visible state. The inverse process acts exactly in the same way. The cross section for such a transformation is proportional to the usual elastic cross section and to the swapping probability $p$ [2]. Following this line of thought, neutron disappearance/reappearance toward/from a hidden brane can then be tested thanks to low-noise experiments [2, 4]. A first experiment of this kind was carried out at the ILL (France) in 2015 [2] making possible to get the following constraint on the neutron swapping probability [3]:

$$
p<4.6 \times 10^{-10} \text { at } 95 \% \mathrm{CL} \text {. }
$$

\section{The MURMUR detector}

MURMUR is a detector currently localized near the BR2 nuclear reactor (at Mol in Belgium) and aiming at constraining some braneworld scenarios [4]. The configuration of the experiment on-site near the BR2 nuclear core is shown in Fig. 3 and a sketch of the MURMUR detector is shown in Fig. 4. When the high thermal neutron flux produced inside the core interacts with the moderator made of beryllium, a certain proportion of the neutrons could be converted into hidden neutrons, see Fig. 3a. While the neutron swapping probability $p$ is expected to be very weak, a high neutron flux is required in order to observe this phenomenon. A nuclear reactor where a high neutron flux can undergo many elastic scatterings in the core-moderator assembly is thus an ideal environment to perform such an experiment. Hidden neutrons emitted from the moderator of the core are then free to propagate out of both the reactor biological shielding and the shielding of the MURMUR detector (a $3.6 \mathrm{~cm}$ thick boron carbide (B4C) box which isolates the whole detector from the visible neutron background). Right after the shielding, a matrix of lead acts as a regenerator. It can make possible to regenerate a part of the hidden neutrons into visible neutrons to be finally detected by a ${ }^{3} \mathrm{He}$ proportional counter. Lead appears to be a good material when dealing with hidden neutron regeneration thanks to its high scattering cross section and as it thermalizes neutrons very poorly. Indeed, some fast or epithermal neutrons could pass through the shielding. As the neutron capture cross section of the ${ }^{3} \mathrm{He}$ increases with decreasing neutron energy, neutrons thermalized in the regenerator would be a source of background. Another source of background are the muon-induced neutrons produced directly in the matrix of lead. A plastic scintillator on the top of the detector 
(a)

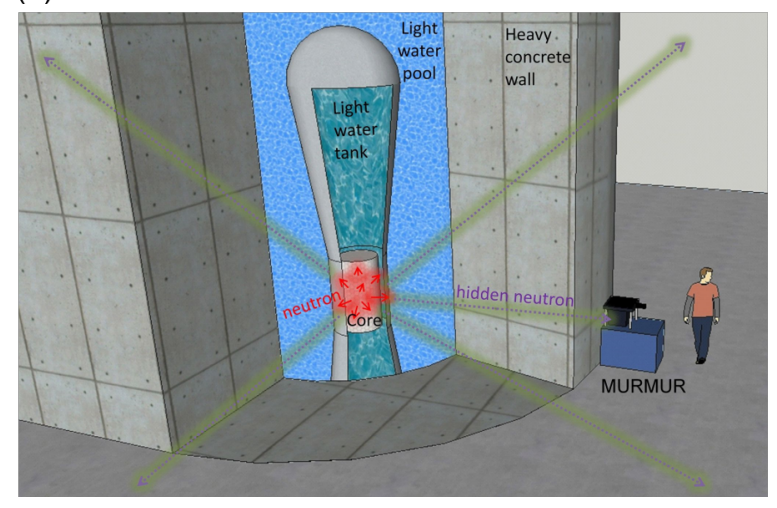

(b)

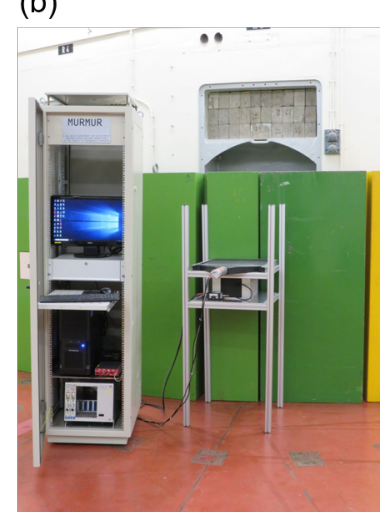

Figure 3: (a) Sketch of the MURMUR experiment near the BR2 core in the SCK.CEN at Mol in Belgium. (b) Photograph of the MURMUR experiment near the BR2 at the SCK.CEN. Paraffin (green walls) have been added between the concrete wall surrounding the reactor and MURMUR in order to suppress the neutron background coming from the core during run periods of the reactor.

used as a veto makes possible to mitigate this source of background. The hidden neutron flux $\Phi_{h}$ at a distance $r$ from the center of the core can be calculated with the following expression:

$$
\Phi_{h}(\mathbf{r})=\frac{p}{8 \pi} \int_{B e} \frac{\Sigma_{S, B e} \Phi_{v}\left(r^{\prime}\right)}{\left|\mathbf{r}-\mathbf{r}^{\prime}\right|^{2}} d r^{3^{\prime}},
$$

where $p$ is the neutron swapping probability, $\Sigma_{S, B e}$ the macroscopic scattering cross section of beryllium and $\Phi_{v}$ the visible neutron flux coming from the core. The neutron rate in the regenerator $\Gamma_{R}$ is given by:

$$
\Gamma_{R}=\frac{p}{2} \Sigma_{S, P b} \Phi_{h} V_{P b},
$$

where $\Sigma_{S, P b}$ is the macroscopic scattering cross section of lead and $V_{P b}$ the total volume of lead. The neutron counting rate in the detector is finally given by:

$$
\Gamma_{\text {Det }}=\xi \Gamma_{R},
$$

with $\xi$ the regenerated neutron detection efficiency, which takes into account the geometry of the experiment and the intrinsic detection efficiency of the neutron detector. The detected neutron rate $\Gamma_{R}$ is thus proportional to $p^{2}$ hence the interest to have a high neutron flux at the beginning. The major improvements of this detector are a regenerator separated from the detector and made of 50 $\mathrm{kg}$ of lead, as well as a better background mitigation, i.e. a subtraction of the background thanks to ON and OFF reactor measurements, a Pulse Shape Discrimination (PSD) acquisition system and an active veto.

\section{First results}

Tab. 1 summarizes the first results of the MURMUR experiment. These results make possible to extract the following counting rate after background subtraction (the OFF-counting rate chosen for 


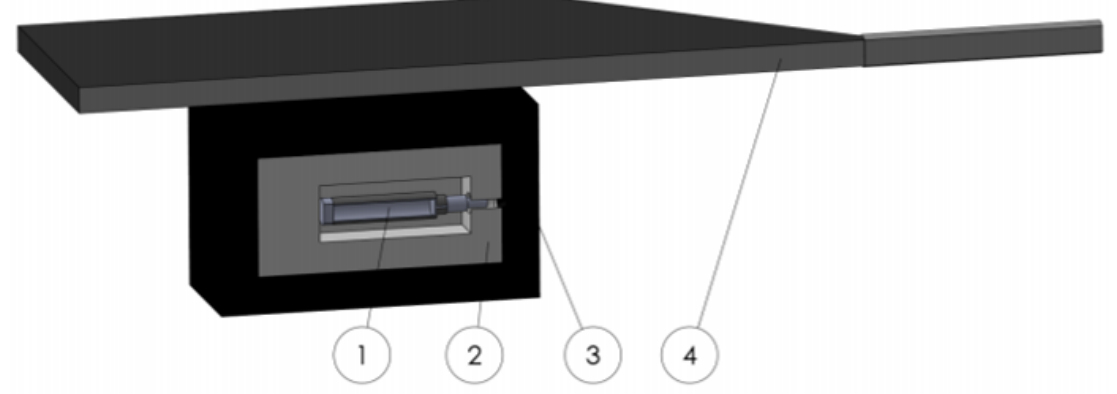

Figure 4: Design of the MURMUR detector. 1. $33.5 \mathrm{~cm}^{3}{ }^{3} \mathrm{He}$ proportional counter. 2. Regenerator: $23.6 \times 17.7 \times 11.5 \mathrm{~cm}^{3}$ of lead $(50 \mathrm{~kg}) .3$. B4C box of $3.6 \mathrm{~cm}$ of thickness. 4 . Plastic scintillator acting as a veto.

\begin{tabular}{lll}
\hline (a) OFF1 $\times 10^{-4} \mathrm{~s}^{-1}$ & (b) $\mathrm{ON} \times 10^{-4} \mathrm{~s}^{-1}$ & (c) $\mathrm{OFF} 2 \times 10^{-4} \mathrm{~s}^{-1}$ \\
\hline $3.09_{-0.16}^{+0.17}$ & $3.10_{-0.17}^{+0.18}$ & $3.09_{-0.15}^{+0.15}$ \\
\hline
\end{tabular}

Table 1: Neutron counting rate in the ${ }^{3} \mathrm{He}$ counter. (a) 831 hours of acquisition during the shutdown period (OFF) of the BR2 nuclear core in December 2018/January 2019. (b) 760 hours of acquisition during the Cycle 02/2019A in April 2019. (c) 998 hours of acquisition during the shutdown period in May/June 2019.

the subtraction is the mean of the two shutdown periods from Tab. 1):

$$
\Gamma_{\text {Det }}<3.3 \times 10^{-5} s^{-1} \text { at } 95 \% \mathrm{CL} \text {. }
$$

The constraint on the swapping probability $p$ is then given by:

$$
p<\sqrt{\frac{\Gamma_{D e t}}{\xi(E) d(E)}}
$$

with

$$
d=\frac{\Sigma_{S, P b}}{16 \pi} \int_{B e} \frac{\Sigma_{S, B e}(E) \Phi_{v}\left(r^{\prime}, E\right)}{\left|\mathbf{r}-\mathbf{r}^{\prime}\right|^{2}} d r^{3^{\prime}}
$$

leading to:

$$
p<4.0 \times 10^{-10} \text { at } 95 \% \mathrm{CL}
$$

This result is only slightly better than the result obtained in 2015, see Eq. 2, and must be understood in the context of the BR2 nuclear reactor core being by a factor of 7.4 less efficient to produce hidden neutrons with respect to the nuclear reactor core of the ILL. Lead as regenerator material and noise subtraction both make possible to give a similar constraint as the previous measurement performed at the ILL. 


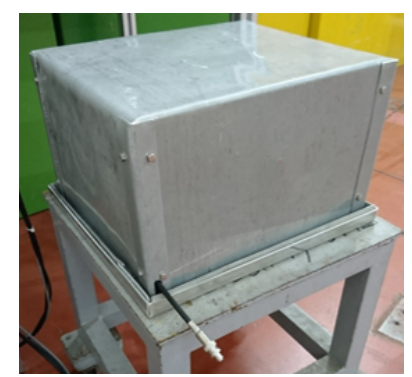

Figure 5: MURMUR detector surrounded by a 2-mm-thick cadmium extra shielding. Measurements with this improved setup are planned at the end of 2020.

\section{Perspectives}

MURMUR is expected to operate at the BR2 until 2021 making possible to test some other experimental configurations. In particular, measurements with extension of the shielding are planned at the end of 2020. The addition of 2-mm-thick cadmium box surrounding the B4C shielding has been made in order to ensure the absorption of epithermal neutrons, see Fig. 5. The addition of a $20-\mathrm{cm}$-thick high density polyethylene is also under consideration in order to thermalize fast neutrons and ensure their absorption by the cadmium and B4C shieldings. The extension of the active veto by the sides of MURMUR is scheduled to further mitigate muon-induced neutrons in the matrix of lead.

\section{References}

[1] M. Sarrazin and F. Petit. Equivalence between domain-walls and "noncommutative" twosheeted spacetimes: Model-independent matter swapping between branes. Phys. Rev. D 81 (2010) 035014.

[2] M. Sarrazin, G. Pignol, J. Lamblin, F. Petit, G. Terwagne, V. V. Nesvizhevsky. Probing the braneworld hypothesis with a neutron-shining-through-a-wall experiment. Phys. Rev. D 91, 075013 (2015).

[3] M. Sarrazin, G. Pignol, J. Lamblin, J. Pinon, O. Méplan, G. Terwagne, P-L. Debarsy, F. Petit, V. V. Nesvizhevsky. Search for passing-through-walls neutrons constrains hidden braneworlds. Phys. Lett. B 758 (2016) 14.

[4] C. Stasser, M. Sarrazin and G. Terwagne. Search for neutron-hidden neutron interbrane transitions with murmur, a low-noise neutron passing-through-walls experiment. EPJ Web of Conferences, 2019.

[5] C. Stasser and M. Sarrazin. Sub-GeV-scale signatures of hidden braneworlds up to the planck scale in a SO(3, 1)-broken bulk. Int. J. of Phys. Mod. A 34(05):1950029 (2019). 